OPEN ACCESS

Edited by:

Raina Robeva,

Randolph-Macon College,

United States

Reviewed by:

Axel Hutt,

Inria Nancy-Grand-Est Research

Centre, France

Miguel Pineda

University College London,

United Kingdom

*Correspondence:

Enrico Capobianco

ecapobianco@med.miami.edu

Specialty section:

This article was submitted to

Dynamical Systems,

a section of the journal

Frontiers in Applied Mathematics and

Statistics

Received: 18 April 2020

Accepted: 25 May 2020

Published: 09 July 2020

Citation:

Capobianco E (2020) Inference From Complex Networks: Role of Symmetry and Applicability to Images.

Front. Appl. Math. Stat. 6:23

doi: 10.3389/fams.2020.00023

\section{Inference From Complex Networks: Role of Symmetry and Applicability to Images}

\author{
Enrico Capobianco * \\ Institute of Data Science and Computing, University of Miami, Coral Gables, FL, United States
}

Symmetry is a mathematical concept only partially explored in networks, especially at the applicative level. One reason is a certain lack of interpretable inference obtained from networks. While the network systemic associations (links) between entities (nodes) emerge from the underlying dependence structure, this latter is only partially explicit via the established direct interactors and remains to a certain extent latent (distant node predicted paths). Verifiability of significant hubs, connectors, paths, and modules allows to build a knowledge base useful to infer latencies and/or validate complex associations. When symmetry is searched in images, reflection, translation and rotation are applicable transformations in $n$-dimensional Euclidean space that computational algorithms target. There is symmetry when original and transformed images cannot be distinguished. Once collected together, such transformations form an automorphism group, indicating a stable and robust global characteristic. It is common to step from images to quantifiable features for conducting inference. Deep learning is typically used to classify whole images reconstructed from the myriads of features in which these images are decomposed. However, with images considered at multiple scales and locations, symmetries are valuable for describing local characteristics. Casting local features into a network framework enables their associations to be explored by similarity or dissimilarity criteria. This is quite intriguing because network configurations may display topological features and connectivity patterns associated with synchronization and symmetry that reduce the redundancy of features to more compact functional descriptions. Then, identifying anomalies from unusual events, behaviors, patterns would spot network vulnerabilities and signs of symmetry breaking.

Keywords: networks, entropy, symmetry, controllability, synchronization, deep learning

\section{INTRODUCTORY REMARKS AND METHODOLOGICAL BACKGROUND}

In physical systems, symmetry commonly means invariance. Therefore, this is like to say that a system looks the same from different observation angles. Equivalently, due to the presence of intrinsic regularities, a system's characteristic may be an observable output in correspondence with multiple interrelated inputs. As symmetries influence the system's functionality and models allow the interpretation of the function dynamics, a general concern is model misspecification. This implies that an inference model $m$ applied to sample data $x \in X$ may fail due to the wrong 
choice of model family $M(x, \theta)$ characterized by parameters $\theta \epsilon \Theta$ and likelihood function $L(\theta \mid x)$. For example, the identifiability of parameters may be violated. Among other possible reasons, parameter non-identifiability may occur due to the presence of symmetry [1].

Formally, symmetry indicates a type of invariance such that a mathematical object remains unchanged under a set of transformations. For instance, a symmetry of a function is a transformation of the function that leaves its graph unchanged. More in general, a symmetry maps an object onto itself, thus preserving its structure. In mathematical terms, a symmetry s: $\theta \rightarrow \theta$ is a measurable function that makes $\theta$ in a model no longer identifiable from the given data $x$. The natural way to obviate to this effect of symmetry is to re-parameterize the model, i.e., $\theta \rightarrow \psi$, with $\psi \in \Psi$. Alternatively, one can constrain the parameters, i.e., $\theta \rightarrow \theta^{c}$. In such cases, the recourse to "symmetry breaking" solutions can be needed to augment the performance of inferential algorithms. Symmetry breaking implies that small system's fluctuations vary beyond established thresholds and that the system may cross a critical point due to the variation governing its state dynamics. Overall, this determines the system's fate because once such transition occurs, it may orient the system toward a different state compared to the initial one.

There are several types of symmetries, the most common being permutations. Some symmetries are local and grouping them leads to transformations that change the system in different ways at different places in space and time. Instead, a symmetry is global if it acts similarly at every point. The fact that for a global symmetry the corresponding parameter is constant explains why the transformation is applied uniformly across the entire system, unlike with a local symmetry in which its parameter is a function of position, and the transformation is applied differently at different points in space and time.

In the system of interest here, i.e., a network $N$, symmetry depends on the preferential attachment growth rule inducing a bias toward multiple short branches that tend to repeat themselves in a tree more than longer ones, and the definition of symmetry is inherent to nodes and their permutations [2]. Given the $N$ adjacency matrix $A$, in which $A_{i j}=1$ (nodes $i, j$ are adjacent) and $O$ (otherwise), a symmetry is present when a permutation $P$ is applied to $A$, leaving it unchanged, i.e., $P A=A$. This so-called automorphism $(A u t)$ means that nodes are topologically equivalent if their permutation does not affect the network structure. This offers a rationale for considering network redundancy and its reduction into network quotients or skeletons [3-5]. Here, structural network properties, including heterogeneity and complexity, remain while repetitions are excluded.

Symmetries may also be combined, but of interest are especially the minimal ones calling all participating nodes to form a symmetric structure, or an orbit $O$ (in which a set of nodes is mapped onto each other). Formally, given $I(N)$ as a set of nodes in $N$ forming a group $A u t(N)$, the orbit of the node $i \in I(N)$ is a set $O(i)=\{\pi \bullet i \in I(N): \pi \in A u t(N)\}$ (see [4]). Thus, nodes belonging to an orbit are intrinsically related by simple permutations in $A u t(N)$. A symmetric network partitioning of its nodes into orbits is thus establishing disjoint equivalence classes for each node, which forms an automorphism partition (Aut-P) [6]. Of interest is also its entropy, which is a measure of the network structural heterogeneity defined as:

$$
E^{A u t-P}=-\sum_{k} p_{k} \log p_{k}
$$

with $k=1, K$, for $K=\operatorname{dim}(A u t-P)$, and with $\mathrm{p}_{\mathrm{k}}$ the associated probabilities (computed as node ratios between Aut- $P$ and network). The normalized entropy is then defined as $E_{N}^{A u t-P}=$ $E^{A u t-P} / \log K$.

It is important to consider a system in steady state (equilibrium) vs. possible departure from it. Such dynamics are regulated by the spectral characteristics. In particular, the eigenvalues of a symmetric structure are decomposable into two types: redundant and non-redundant. Redundancy is in correspondence with the eigenvectors localized on the symmetric structure. The non-redundant eigenvectors refer to eigenvalues with the same values relatively to the nodes of a given orbit. This is relevant for the purpose of reducing the network to the consideration of orbits instead of nodes, which eliminates the redundancy. Conversely, this is a structural property of symmetric networks.

Networks are also a paradigm of complexity. If we insert a probability measure $P^{N}$ in a network $N$, which defines a probability distribution $\operatorname{Pr}(N)$ on its nodes, each having a probability $p r_{i}$, we can also define a Network Entropy as:

$$
E^{N}=\min \sum_{i} p r_{i} \log p r_{i}
$$

This entropy measures the randomness degree in the network and implies the importance of stochasticity, with a role played by symmetries too. Namely, measures may vary depending on fuzzy symmetries [7] (based on fuzzy measure theory) and stochastic symmetries [8] (based on network ensembles). Furthermore, network communities (defined as cohesively connected sets of functionally similar nodes) exert strong inference impacts, although there are cases in which functional node similarity refers to symmetry rather than community effects. A typical example of such ambiguity is provided by studies of brain areas.

The role of symmetries is especially relevant with reference to network synchronization (see [9]), and computational methods to break such symmetries (isolated desynchronization) are discussed in [10]. The construction of functional networks depends on the relationships between their coupling components, which makes synchronization motifs central features. Functional networks are in general heterogeneous or non-symmetrical structures. This reflects the fact that disruption of the couplings generates symmetry-breaking in the network, and also loosens the inherent distribution of synchronization motifs. However, if the couplings are able to sustain synchronization, then symmetry will be characterizing the functional network [11].

Finally, controllability refers to the ability of a dynamical system to step between states, say from an initial to a final state, 
in finite time $[12,13]$. This possibility can be verified quite straightforwardly in linear time-invariant systems by the socalled Kalman's rank condition. In particular, given a canonical system $d z=A z+B v$, with state vector $z$, and $A$, and $B$ as state and control parameter matrices, respectively, the requirement is: $\operatorname{rank}\left[B, A B, \ldots \ldots, A^{P-1} B\right]=P$. Naturally enough, a complication is when the system's parameters are unknown. Furthermore, a common strategy to establish network controllability turns to the identification of a minimal set of driver nodes. This implies that controlling this set allows to exert control over the entire system [14].

With reference to symmetry, what reported in [15] is relevant for the focus on network controllability but considering the impact of individual rather than global dynamics, thus departing from network topology. In real-world networks, assumptions like the independence of parameters must be relaxed to face the presence of dependence and even interdependence structure. Thus, a global symmetry aspect is a characteristic to consider in assessing both single and combined networks, and establishing the highest possible controllability, i.e., the lowest number of critical nodes, becomes a primary goal in applications. Intuitively, specific hub or module dynamics may remain locally informative but become less relevant for controllability scopes compared with the densities at which they present at network scale, which reflects their possible influence. Figure $\mathbf{1}$ reconciles the main concepts in this section.

Nevertheless, real networks complexity may be reduced by considering node-specific hidden variables that once transformed may reveal latent symmetries. Such transformations may extend to statistical ensembles designed through families of stochastic networks [16]. For the networks partition $N=\left[N_{1}, N_{2}, \ldots, N_{Q}\right]$ and the associated probabilities assigned to each of them $\operatorname{Pr}(\mathrm{N})$ $=\left[\operatorname{Pr}\left(N_{1}\right), \operatorname{Pr}\left(N_{2}\right), \ldots \operatorname{Pr}\left(N_{Q}\right)\right]$, it holds that $\sum_{N} \operatorname{Pr}(\mathrm{N})=\sum_{q}$ $\operatorname{Pr}\left(N_{q}\right)=1$ for $q=1, Q$. These networks will be stochastically symmetric under a transformation if each member network has the same properties under the transformation. An associated entropy optimization problem is the one searching for the $\operatorname{Pr}(\mathrm{N})$ that maximizes the Shannon-Gibbs entropy:

$$
E^{S G}=-\sum_{N} \operatorname{Pr}(N) \log \operatorname{Pr}(N)
$$

This problem usually involves a topologically constrained network, particularly when the ensemble network functions as a null model (more details on probability functions and entropies can be found in [8]).

\section{COMPUTATIONAL ASPECTS OF NETWORK SYMMETRY}

Symmetry exerts several types if influences (see Figure 2). A first main question generally addressed is how to quantify the redundancy that is due to the presence of symmetry in a network. One way is through the compression ratio [17], a measure that compares the full network $\mathrm{N}$ to its quotient $\mathrm{Q}$ (counting one node per orbit) computed in two possible ways as either:

$$
C^{1} r=\left(n_{N} / n_{Q}\right)^{2}
$$

where the ratio is between the number of nodes $n_{N}$ and the number of orbits $n_{Q}$ in the full network, or

$$
C^{2} r=l_{N} / l_{Q}
$$

this time with edges $l_{N}$ and $l_{Q}$ (representing average connectivity) used in a sparse network.

It is between these two measures that the redundancy of an arbitrary network can fluctuate. A direct compression of symmetry is also possible, through the quotient matrix $Q(A)$, obtained from the adjacency matrix $A$ and the characteristic matrix $S$ (i.e., referred to the network partition associated to the quotient):

$$
Q(A)=S^{T} A S
$$

Importantly, symmetry leaves also a spectral signature through the presence of peaks in the spectral density, to which redundant eigenvectors are associated, i.e.,

$$
\rho(\lambda)=1 / N \sum_{i=1, N} \delta\left(\lambda-\lambda_{i}\right)
$$

or a sum of Dirac delta functions with $\lambda_{i}$ as the largest eigenvalue [4]. When the spectrum of a network adjacency matrix is considered, symmetries differentiate according to whether a symmetric structure is present, for then the eigenvalues can be decomposed into two classes: a) with redundant eigenvalues, in correspondence to eigenvectors localized on the symmetric structure and $b$ ) with non-redundant eigenvalues, i.e., whose eigenvectors have identical values in all elements that correspond to nodes in the same orbit. As an alternative, similar results were obtained for the Jacobian matrix in a food web model [18].

Looking beyond partitions from nodes to orbits, one can consider equitable partitions in which two node clusters have a special relationship, one in which each node of one cluster has exactly the same number of neighbor nodes in the other cluster. Interestingly, this complementary partition has relevance for synchronous patterns, as in both types of partitions nodes in the same cluster can synchronize and in different clusters cannot [19].

It is consolidated nowadays as a practice the fact to extensively use Deep Learning (DL) in image applications. It is rarer to find symmetry at the core of studies, despite the relevance [20]. An interesting study [21] of generative adversarial networks (GAD) has exploited horizontal symmetry (usually not considered in such form) by adopting two methods, one checking what symmetry loss allows generated images and flipped versions to be classified the same way, and another that inverts the flipped images aiming at reconstructing with minimal distortion. Another convolutional neural 


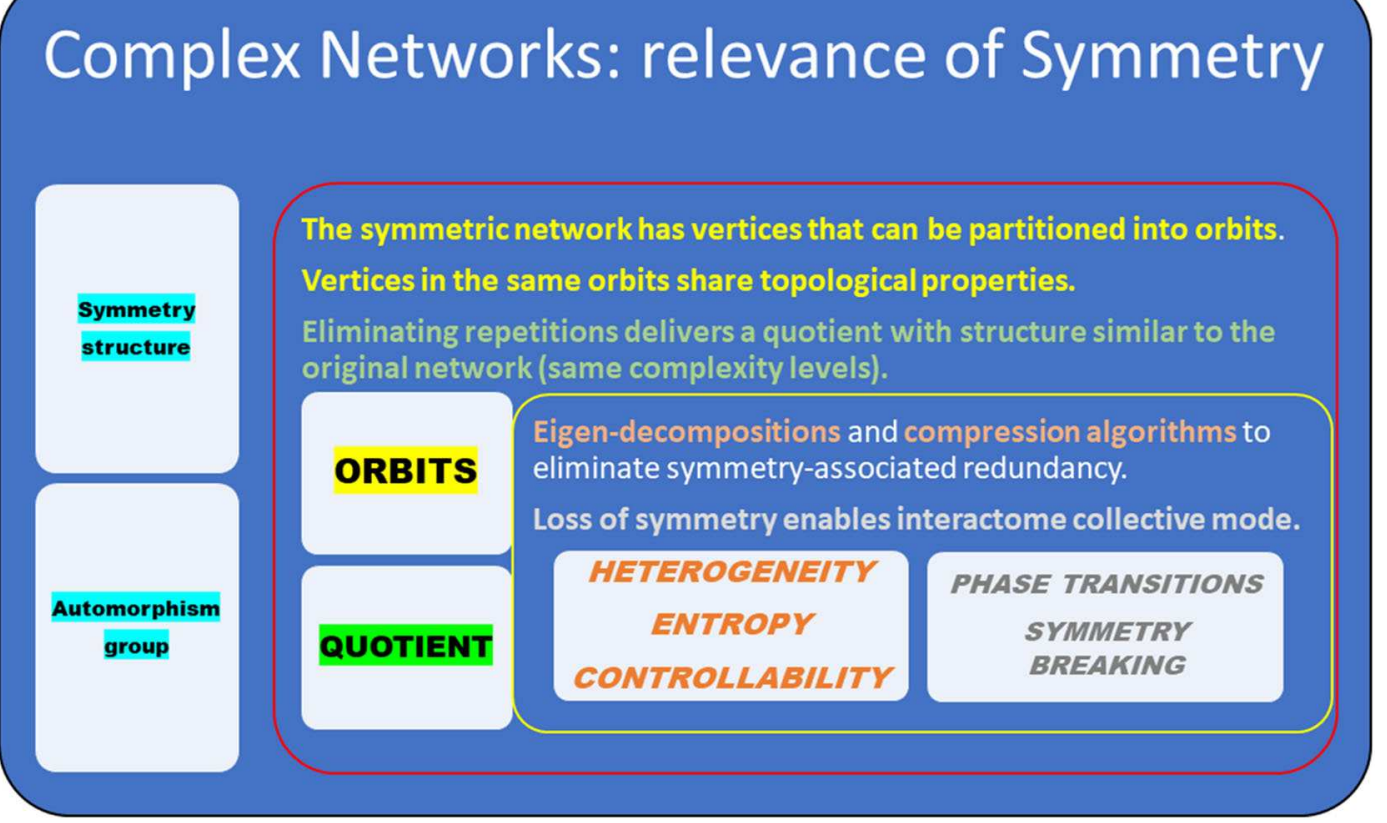

FIGURE 1 | Hierarchy of symmetry relationships in networks.

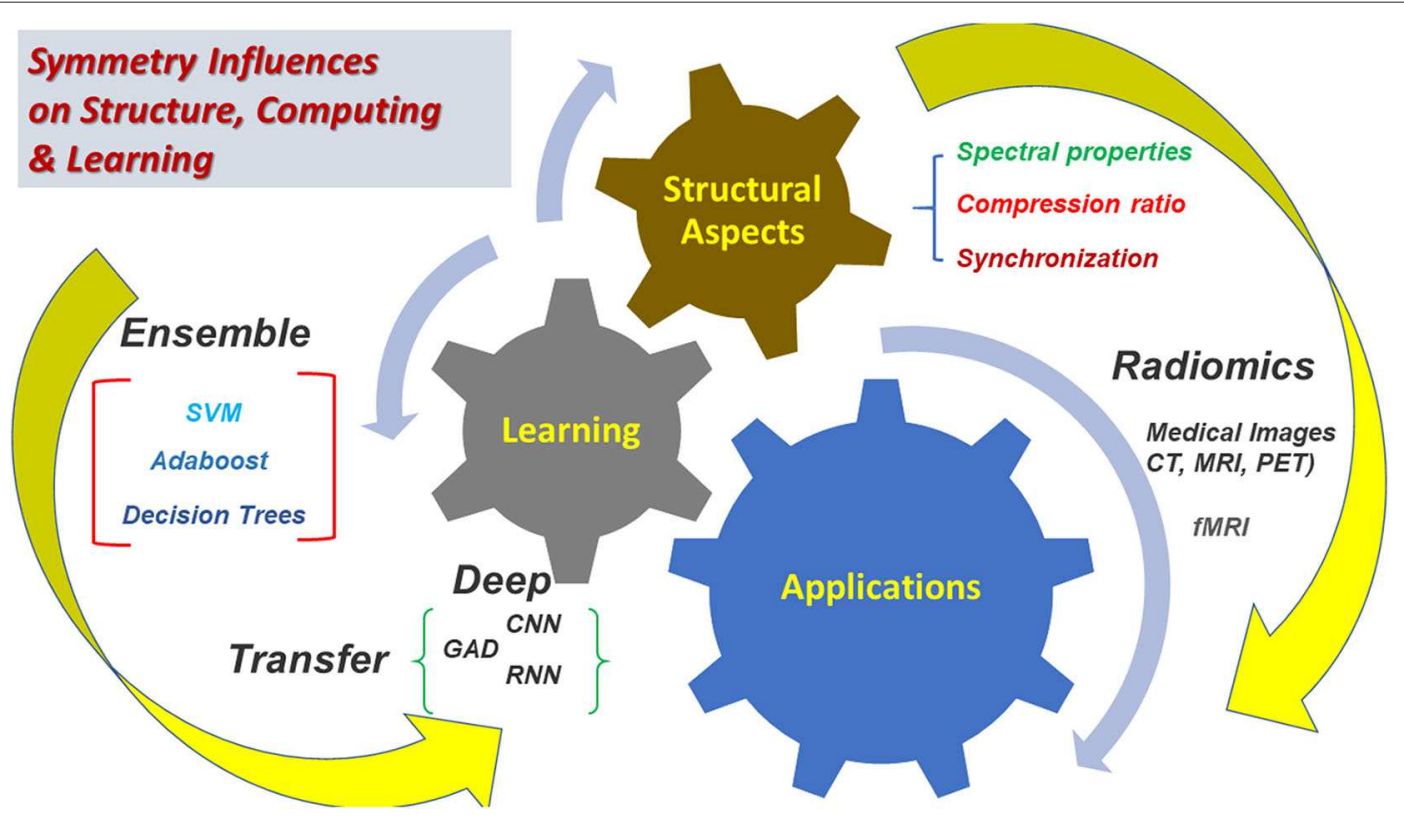

FIGURE 2 | Symmetry outreach.

neural networks (CNN) study [22] inspired by primary visual cortex processes has investigated the impact of symmetry constraints in convolutional layers for image classification. As a result, similar performance was found in a setting with reduced number of parameters due to replacement of random weights by symmetry constraints during backpropagation.
Interestingly, the imposition of symmetry constraints to reduce the number of parameters was assessed in another study [23] in which accuracy loss was absolutely limited even in overparameterized settings (both $\mathrm{CNN}$ and recurrent neural networks or RNN) from CIFAR, ImageNet etc. As a general remark, data paucity and non-standard statistical settings (non-independently identically distributed, unsupervised etc.) 
have suggested to embed learning architectures with invariance under symmetry transformations. In a study [24] on probabilistic symmetry, a link with functional symmetry was established such that the structure of networks could be completely characterized by invariance usable to construct both stochastic and deterministic solutions.

Different applications have been presented by two further studies. One study [25] was centered on a spreading model in a setting of multilayer networks that considers non-equilibrium phase transition when either susceptible-infected-susceptible or susceptible-infected-recovered spreading dynamics being integrated. Using a biased diffusion process among different layers, the discontinuous transition goes together with a spontaneous symmetry breaking in occupation probabilities of individuals in each layer. Another interesting study [26] was proposed on the symmetry of weighted brain networks to decipher the roles of individual brain areas and the redundancy of connectivity. The structural symmetry of every pair of nodes in the network was quantified by the isomorphism of the residual graphs. Then, fMRI was performed on subjects with a condition, i.e., inattentive type of Attention Deficit Hyperactivity Disorder, showing the emergence of higher level of network symmetry compared to the development group. As a methodological note, symmetry levels threshold-sensitivity was observed.

\section{IMAGE NETWORKS AND ROLE OF SYMMETRY}

Radiomics [27-29] promotes the role of images as mineable data [30]. These are features providing the medical imaging community with a wealth of quantitative information usable for diagnosis, therapy evaluation, patient risk profiling and stratification. The literature of radiomics is dense of applications centered on pipelines that leverage machine learning (ML) implementation of feature selection and image fusion methods. Especially the DL community found in radiomics a precious source of data and a testing ground for ML and statistical methods. In turn, the advent of this discipline has stimulated increasingly integrative research on cancer biology, genomics and clinics with unprecedented scale and resolution depth [31]. Thus, it has become possible to investigate the complex hierarchical organization of medical images.

The key factor is designing DL and ML solutions targeted to a synthesis of various types of measurements and predictions, either assembled together in the attempt to concentrate information or distributed across network layers in order to reconcile all the differently channeled information into scores. These two types of approach, concentric one and distributed the other, may present quite different symmetry scenarios, despite a differentiation occurs according to the achievable precision, thus depending on imaging technologies and combination of modalities together with data gaps.

From a modeling standpoint, and by keeping symmetry in mind, transfer learning (TL) [32-36] and ensemble modeling (EM) seems especially relevant. The TL's paradigm is that features may be learned in a certain application domain and then applied to different domains. The role of symmetry could be an important criterion to judge generalizability first and feasibility after, for then leaving to the ability of TL leveraging other similarity aspects (related or not to symmetry). EM seems to match well the distributed approach discussed earlier by suggesting strategies for weighting scores and predictions from different models. However, data features gathered at a variety of spatiotemporal resolutions are hard to integrate into interpretable predictive models. Quite evidently, there is strong need to identify and measure heterogeneity in spatial and time, and then crosscorrelate all types of imaging features.

Structural symmetry is central to human brain functions and thus the functional activities of areas that are symmetric at an anatomic level can be strongly correlated, even if the areas are distant in space. Experiments in [9] studied symmetry in the human brain via coupled anatomical (DW-MRI data) and functional (fMRI) connectivity graphs defined on the same set of 90 cortical areas. Interestingly from a network standpoint the fact the relevance of anatomical symmetry in neural synchronization because determining correlated functional modules across distant locations. At one end anatomically symmetric regions obtained via clustering of nodes with phase at stationary state, at the other end a functional network with links indicating statistically significant correlations between the timeseries of cortical areas. For two nodes in spatially separated regions showing no anatomical connection it appeared symmetry and strong synchronization from fMRI. For other two nodes from spatially adjacent regions and anatomically connected a gap in phase emerged together with lack of fMRI synchronized patterns.

In an interesting study [37], non-contrast (nc) CT images with follow-up magnetic resonance diffusion-weighted (MR-DW) images have supported evidence on the differentiation between ischemic and healthy brain tissue through quantification of symmetry. Stroke spots were identified by spatially aligning MRDW images to the corresponding ncCT images. An interesting observation is that three methods (AdaBoost, Support Vector Machines, Decision Trees) were used to classify, but a basic limitation in terms of accuracy remained when comparing the textures only between the stroke lesions while considering as normal the contralateral regions. Despite the symmetrical acute strokes would be uncommon, other ischemic lesions or pathologies might be present in the contralateral region and require selection of features ad hoc for differentiating stroke regions from normal ones.

In the above example networks were not used, but in general our ability to carry or block information through a network depends on its topology. Especially when the conductivity or information transfer is considered then spatial symmetry becomes central together with its density or average number of links per node and topological dimension [38]. Therefore, an alternative method of classification ideally fitting the experiments in would involve encoding equivariance in learning [39]. In order to approximate invariance to a class of transformations of the input, a neural network would need training via with data augmentation (see [40], and related references in this work). With enough capacity, the network may learn such invariances but without guarantee of generalization. 
The specific patterns of synchronization detectable in a network provide information about the underlying couplings dynamics and when considered in all their relationships can well characterize the system under study. Even with nonlinear dynamics interaction regimes become identifiable and the network structure inferable from similarity and connectivity. Coexisting complex synchronized dynamics male a network stable and robust against external factors (stressors/perturbations) and heterogeneous motifs are probes for functional connectivity, particularly when stable as they become an invariant feature (i.e., consistency of synchronization patterns). Thus, the relationship between network symmetries and the consistency of the synchronization patterns, particularly with coupling inducing global synchronization and resulting in symmetrical networks reflected onto functional ones, as shown in the brain context (see [11] for all details and related references).

\section{DISCUSSION}

We keep a final point for discussion. Identifying anomalies from unusual events, behaviors, patterns can be useful to spot network vulnerabilities and may reveal presence of symmetry breaking. Conversely, the control of large dynamical complex networks may depend on the identification of just a few input nodes or modules. Therefore, by keeping in mind what the targets are, the results from exerting control through networks can be quite efficiently achievable, although only approximately.

The identification of symmetries in a complex network remains important in order to decipher its organizational principles and rules. It is key to understand the role of symmetries in reconstructing or controlling network dynamics [41]. It is important to decompose a network in two possible ways: one into observable/controllable vs. unobservable/uncontrollable sub-networks, and another one into symmetry-driven vs. nonsymmetry-driven sub-networks. Finally, it is key to study how network components synchronize or desynchronize because network functionalization depends on such coupled dynamics. Naturally enough, the problems are always much harder in

\section{REFERENCES}

1. Nishihara R, Minka T, Tarlow D. Detecting parameter symmetries in probabilistic models. arXiv [Preprint] arXiv:1312.5386v1 (2013).

2. Sevim V, Rikvold PA. Effects of preference for attachment to low-degree nodes on the degree distributions of a growing directed network and a simple food-web model. Phys Rev E Stat Nonlin Soft Matter Phys. (2006) 73(5 Pt 2):056115. doi: 10.1103/PhysRevE.73.056115

3. Xiao Y, MacArthur BD, Wang H, Xiong M, Wang W. Network quotients: structural skeletons of complex systems. Phys Rev E. (2008) 78:046102. doi: 10.1103/PhysRevE.78.046102

4. MacArthur BD, Sanchez-Garcia RJ. Spectral characteristics of network redundancy. Phys Rev E. (2009) 80:026117. doi: 10.1103/PhysRevE.80.026117

5. Wang H, Yan G, Xiao Y. Symmetry in world trade network. J Syst Sci Complex. (2009) 22:280-90. doi: 10.1007/s11424-009-9163-9

6. Xiao $\mathrm{YH}, \mathrm{Wu}$ WT, Wang $\mathrm{H}$, Xiong $\mathrm{M}$, Wang W. Symmetrybased structure entropy of complex networks. Phys A. (2008) 387:2611-19. doi: 10.1016/j.physa.2008.01.027 non-linear networks as observability and controllability must deal with more complex dependence relationships.

The last considerations go to the application domain. Symmetry has several impacts on very transformative fields like robotics, computer vision, computer graphics, medical image analysis, radiomics all characterized by coupling artificial intelligence and machine learning with geometry, group theory, graphs, statistics etc. Recognizing symmetries is instrumental to the retrieval of structure from redundant noisy systems, therefore a statistical problem too, which brings in lots of other applications and many possible model frameworks (see for instance examples from network medicine applied to cancer data of various complexities [31]).

\section{DATA AVAILABILITY STATEMENT}

The original contributions presented in the study are included in the article/supplementary materials, further inquiries can be directed to the corresponding author/s.

\section{AUTHOR CONTRIBUTIONS}

The author confirms being the sole contributor of this work and has approved it for publication.

\section{FUNDING}

Support for study \& research activity in network science is acknowledged from NIH AWD-005216 GRANT GR013673 and NSF 19-500 GRANT; DMS 1918925/1922843 (years 2019-22).

\section{ACKNOWLEDGMENTS}

The author thanks colleagues and scholars for deep discussion on these topics at participated seminars and talks. The author thanks two reviewers for their comments on a first draft of the paper. Support for study \& research activity in network science is acknowledged from NIH AWD-005216 GRANT GR013673 NSF-NIH grant DMS-1922843.
7. Garrido A. Symmetry in complex networks. Symmetry. (2011) 3:115. doi: 10.3390/sym 3010001

8. Garlaschelli D, Ruzzenenti F, Basosi R. Complex networks and symmetry I: a review. Symmetry. (2010) 2:1683-709. doi: 10.3390/sym2031683

9. Nicosia V, Valencia M, Chavez M, Diaz-Guilera A, Latora V. Remote synchronization reveals network symmetries and functional modules. Phys Rev Lett. (2013) 110:174102. doi: 10.1103/PhysRevLett.110. 174102

10. Pecora LM, Sorrentino F, Hagerstrom AM, Murphy TE, Roy R. Cluster Synchronization and isolated desynchronization in complex networks with symmetries. Nat Commun. (2014) 5:4079. doi: 10.1038/ncomms5079

11. Malagarriga D, Villa AEP, Garcia-Ojalvo J, Pons AJ. Consistency of heterogeneous synchronization patterns in complex weighted networks. Chaos. (2017) 27:031102. doi: 10.1063/1.4977972

12. Kalman RE. Mathematical description of linear dynamical systems. J SIAM Control Ser A. (1963) 1:152-92. doi: 10.1137/0301010

13. Luenberger DG. Introduction to Dynamic Systems: Theory, Models and Applications. New york, NY: John Wiley \& Sons, Inc., (1979). p. 446. 
14. Liu YY, Slotine JJ, Barabasi AL. Controllability of complex networks. Nature. (2011) 473:167-73. doi: 10.1038/nature10011

15. Zhao C, Wang WX, Liu YY, Slotine JJ. Intrinsic dynamics induce global symmetry in network controllability. Sci Rep. (2015) 5:8422. doi: $10.1038 /$ srep08422

16. Marras E, Travaglione A, Capobianco E. Sub-modular resolution analysis by network mixture models. Stat Appl Genet Mol Biol. (2010) 9:19. doi: 10.2202/1544-6115.1523

17. Garcia RJS. Exploiting symmetry in network analysis. Commun Phys. (2020) 3:87.

18. Aufderheide $\mathrm{H}$, Rudolf L, Gross T. Mesoscale symmetries explain dynamical equivalence of food webs. New J Phys. (2012) 14:105014. doi: 10.1088/1367-2630/14/10/105014

19. Siddique AB, Pecora L, Hart JD. Symmetry and input cluster synchronization in networks. Phys Rev E. (2018) 97:042217. doi: 10.1103/PhysRevE.97.042217

20. Griffin LD. Symmetries of 1-D Images. J Math Imaging Vis. (2008) 31:15764. doi: 10.1007/s10851-008-0078-1

21. Makkapati V, Patro A. Enhancing Symmetry in GAN Generated Fashion Images. Cambridge: Springer International Publishing (2017). p. 40510. doi: 10.1007/978-3-319-71078-5_34

22. Dzhezyan G, Cecotti H. SymNet: symmetrical filters in convolutional neural networks. arXiv. (2019) 187:102786.

23. Hu SX, Zagoruyko S, Komodakis N. Exploring weight symmetry in deep neural networks. Comput Vis Image Und. (2019) 187:102786. doi: 10.1016/j.cviu.2019.07.006

24. Bloem-Reddy B, Teh YW. Probabilistic symmetry and invariant neural networks. arXiv [Preprint] arXiv:1901.06082v1 (2020)

25. An N, Chen H, Ma C, Zhang H. Spontaneous symmetry breaking and discontinuous phase transition for spreading dynamics in multiplex networks. New J Phys. (2018) 20:125006. doi: 10.1088/1367-2630/ aaf660

26. Hu C, El Fakhri G, Li Q. Evaluating structural symmetry of weighted brain networks via graph matching. Med Image Comput Comput Assist Interv. (2014) 17(Pt 2):733-40. doi: 10.1007/978-3-319-10470-6_91

27. Lambin P, Rios-Velazquez E, Leijenaar R, Carvalho S, van Stiphout RG, Granton P, et al. Radiomics: extracting more information from medical images using advanced feature analysis. Eur J Cancer. (2012) 48:4416. doi: 10.1016/j.ejca.2011.11.036

28. Parekh V, Jacobs MA. Radiomics: a new application from established techniques. Expert Rev Precis Med Drug Dev. (2016) 1:207-26. doi: 10.1080/23808993.2016.1164013

29. Gillies RJ, Kinahan PE, Hricak H. Radiomics: images are more than pictures, they are data. Radiology. (2016) 278:563-77. doi: 10.1148/radiol.2015151169

30. Hosny A, Parmar C, Coreller TP, Grossmann P, Zeleznik R, Kumar A, et al. Deep learning for lung cancer prognostication: a retrospective multi-cohort radiomics study. PLOS Med. (2018) 15:e1002711. doi: 10.1371/journal.pmed.1002711
31. Capobianco E, Dominietto M. From medical imaging to radiomics: role of data science for advancing precision health. J Pers Med. (2020) 10:15. doi: 10.3390/jpm10010015

32. Sevakula RK, Singh V, Verma NK, Kumar C, Cui Y. Transfer learning for molecular cancer classification using deep neural networks. IEEE/ACM Trans Comput Biol Bioinform. (2019) 16:2089-100. doi: 10.1109/TCBB.2018.2822803

33. Kensen A, Harrison PJ, Spjuth O. Transfer learning with deep convolutional neural networks for classifying cellular morphological changes. SLAS Discov. (2019) 24:466-75. doi: 10.1177/2472555218818756

34. Xu Y, Hosny A, Zaleznik R, Parmar C, Coreller T, Franco I, et al. Deep learning predicts lung cancer treatment response from serial medical imaging. Clin Can Res. (2019) 25:11. doi: 10.1158/1078-0432.CCR-18-2495

35. Matos Jd, Britto AdS, Oliveira LES, Koerich AL. "Double Transfer Learning for Breast Cancer Histopathologic Image Classification,” In: 2019 International Joint Conference on Neural Networks (IJCNN). Budapest (2019) 1-8. doi: 10.1109/IJCNN.2019.8852092.

36. Yang Y, Yan LF, Zhang X, Han Y, Nan HY, Hu YC, et al. Glioma grading on conventional MR images: a deep learning study with transfer learning. Front Neurosci. (2018) 12:804. doi: 10.3389/fnins.2018.00804

37. Peter R, Korfiatis, P, Blezek D, Beitia AO, Stepan-Buksakowska I, Horinek D, et al. A quantitative symmetry-based analysis of hyperacute ischemic stroke lesions in noncontrast computed tomography. Med Phys. (2017) 44:1929. doi: $10.1002 / \mathrm{mp} .12015$

38. Zhukov DO, Andrianova EG, Lesko SA. The influence of a network's spatial symmetry, topological dimension, and density on its percolation threshold. Symmetry. (2019) 11:920. doi: 10.3390/sym11070920

39. Sander Dieleman S, De Fauw J, Kavukcuoglu K. Exploiting cyclic symmetry in convolutional neural networks. ICML'16: Proceedings of the 33rd International Conference on Machine Learning (New York, NY). (2016) p. 1889-98.

40. Whalen AJ, Brennan SN, Sauer TD, Schift SJ. Observability and controllability of nonlinear networks: the role of symmetries. Phys Rev X. (2015) 5:011005. doi: 10.1103/PhysRevX.5.011005

41. Capobianco E. Next generation networks: featuring the potential role of emerging applications in translational oncology. J Clin Med. (2019) 8:E664. doi: $10.3390 / \mathrm{jcm} 8050664$

Conflict of Interest: The author declares that the research was conducted in the absence of any commercial or financial relationships that could be construed as a potential conflict of interest.

Copyright (c) 2020 Capobianco. This is an open-access article distributed under the terms of the Creative Commons Attribution License (CC BY). The use, distribution or reproduction in other forums is permitted, provided the original author(s) and the copyright owner(s) are credited and that the original publication in this journal is cited, in accordance with accepted academic practice. No use, distribution or reproduction is permitted which does not comply with these terms. 